Supporting Information

\title{
Natural rubber latex reinforced by graphene oxide/zwitterionic chitin nanocrystals hybrids for high-performance elastomer without sulfur vulcanization
}

Chengshun Liut, Shasha Huangt, Jiarui Hout, Wei Zhangł, Jinheng Wangt, Hongsheng Yang ${ }^{*}$, Jianming Zhang*t

tKey Laboratory of Rubber-Plastics, Ministry of Education/ Shandong Provincial Key Laboratory of Rubber-plastics, Qingdao University of Science \& Technology, Qingdao 266042, China.

FCAS Key Laboratory of Nano-Bio Interface, Suzhou Institute of Nano-Tech and Nano-Bionics, Chinese Academy of Sciences, Suzhou, Jiangsu, 215123, China

Number of pages: 12

Number of figures: 9

Number of schemes:0

Number of tables: 1 
Figure S1 Characterization of GO, showing GO is graphene oxide rather than graphite oxide.

Figure S2 AFM images of GC hybrids.

Figure S3 FTIR spectra of GO, NC, and GC, showing hydrogen bonding between GO and NC.

Figure S4 FTIR spectra of NC, NR, and NR/NC, showing hydrogen bonding between $\mathrm{NC}$ and NR particles.

Figure S5-7 Characterization of NR nanocomposites.

Figure S8-9 and Table S1 Characterization of medical gloves. 
(a)

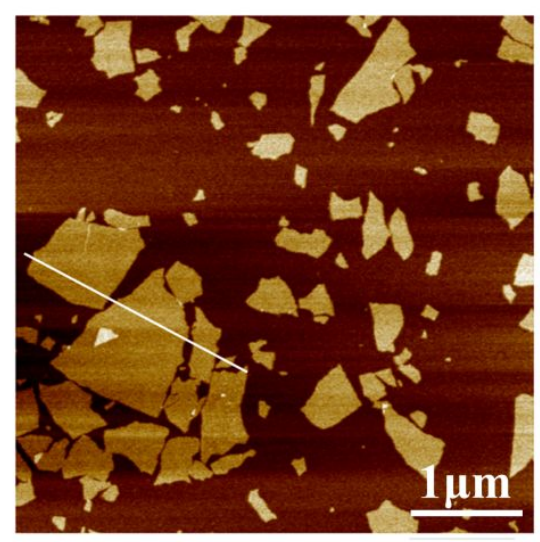

(b)

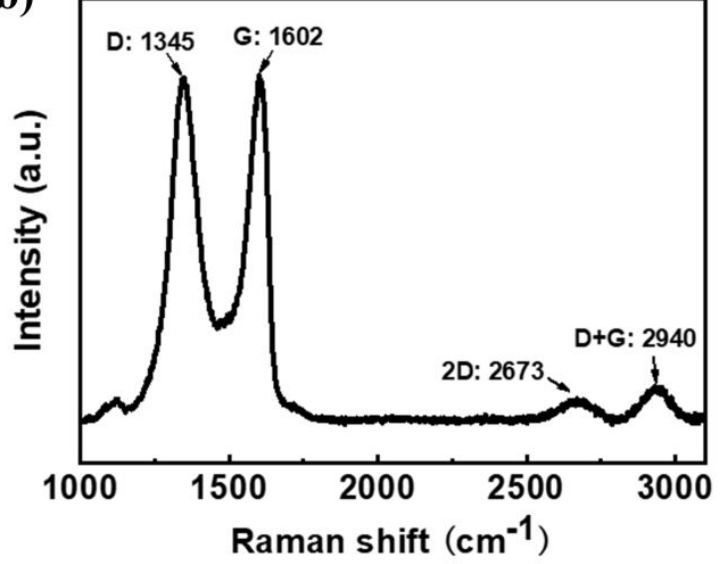

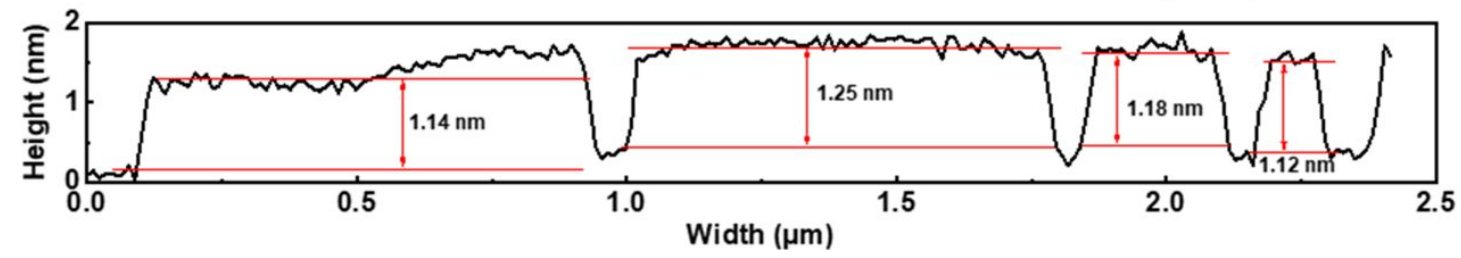

Figure S1. (a) AFM image and height of GO, (b) Raman spectra of GO.

The average thickness of GO nanosheets is about $1.2 \mathrm{~nm}$, indicating the effective exfoliation, as shown in Figure S1a. Beyond that, Figure S1b shows GO exhibits D, G, 2D, and D+G at $1345 \mathrm{~cm}^{-1}, 1602 \mathrm{~cm}^{-1}, 2673 \mathrm{~cm}^{-1}$, and $2940 \mathrm{~cm}^{-1}$, respectively. 
(a)

(c)
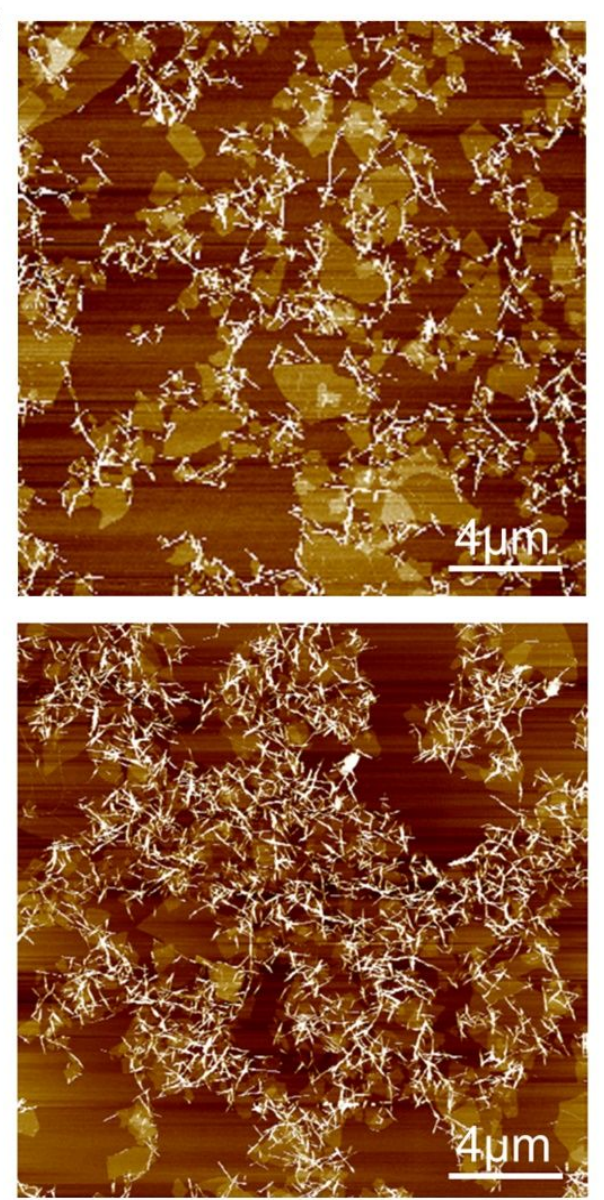

(b)

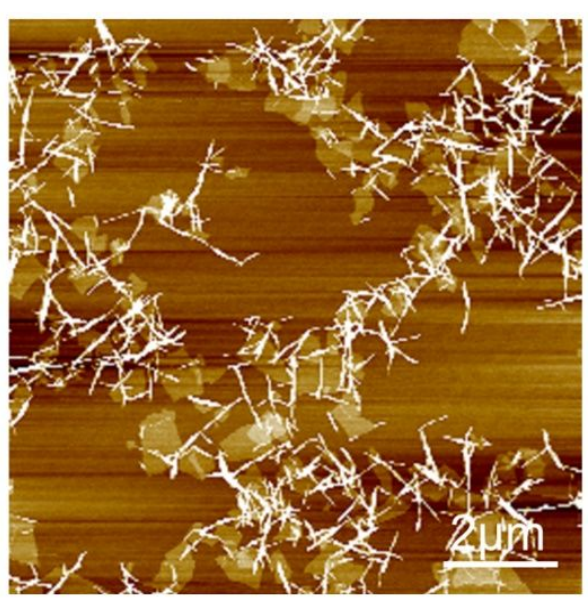

(d)

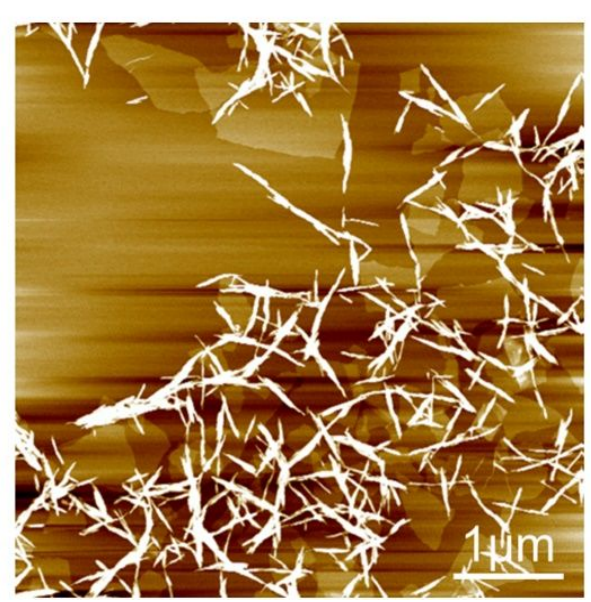

Figure S2. AFM images of GC in different locations. 


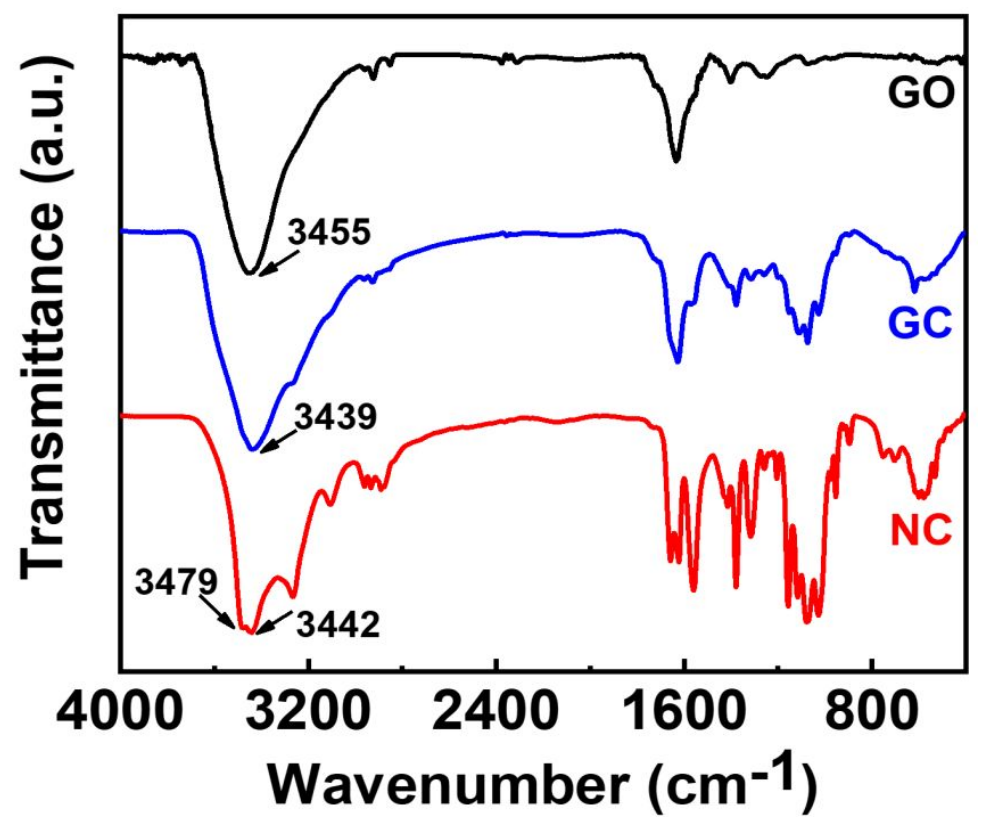

Figure S3. FTIR spectra of GO, NC, and GC. 

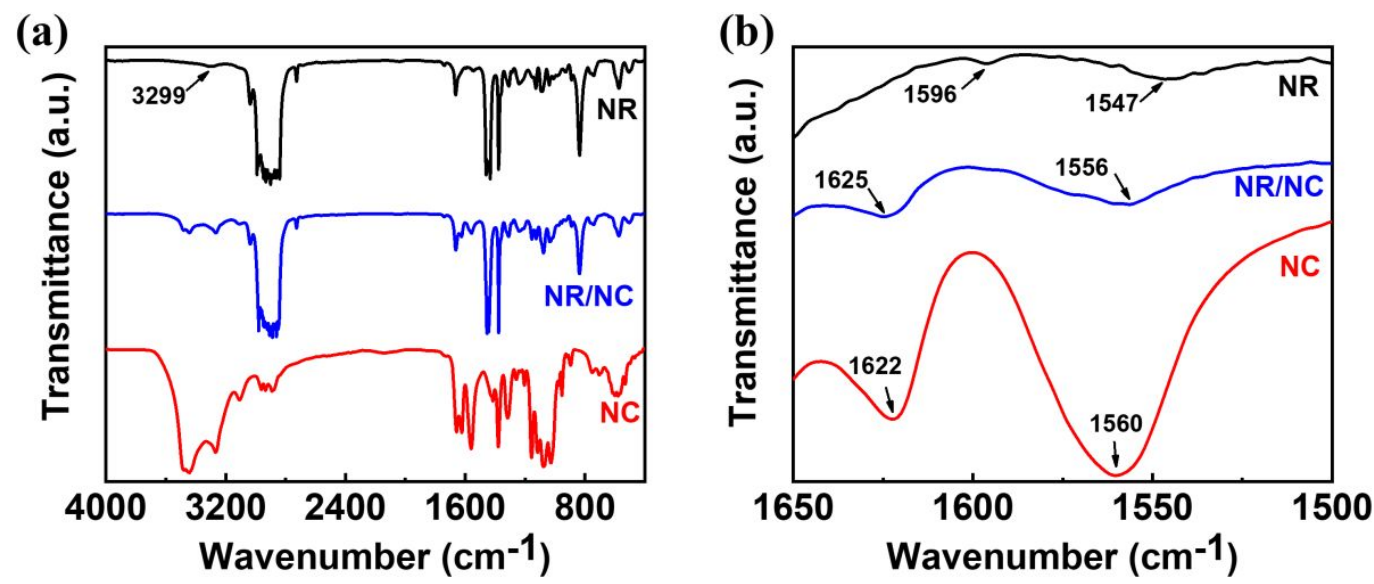

Figure S4. (a) FTIR spectra of NR, NC, and NR/NC; (b) FT-IR spectra of NR, NC, and NR/NC describing the hydrogen bonds between the $\mathrm{NC}$ and the protein of $\mathrm{NR}$ particles. 


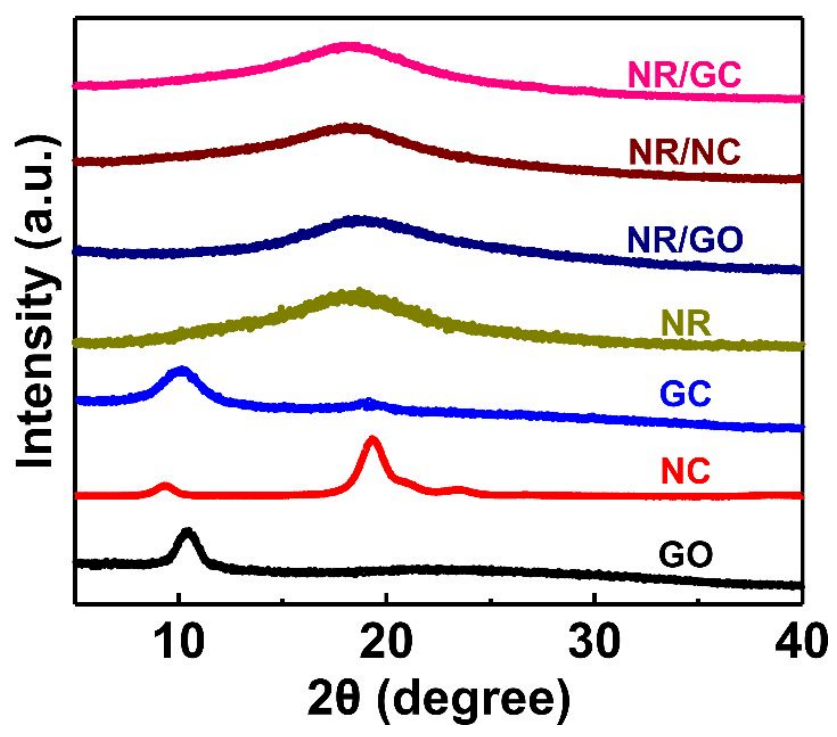

Figure S5. XRD spectra of GO, NC, GC, and nanocomposites. 
(a)

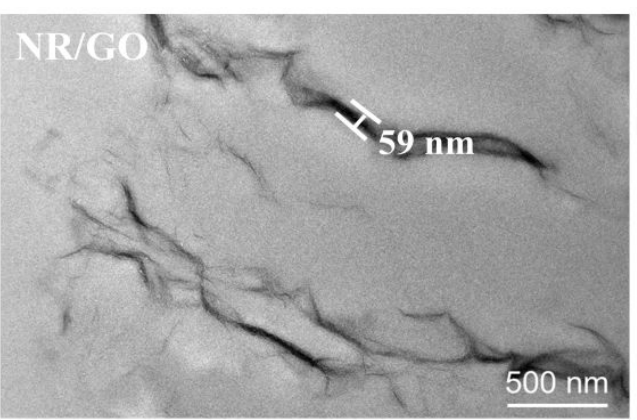

(b)

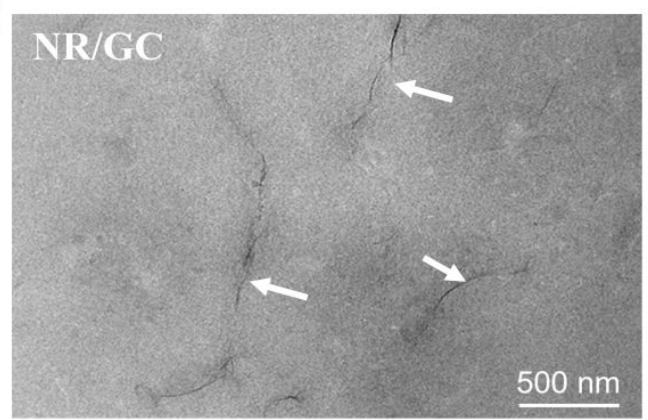

Figure S6. TEM images of (a) NR/GO and (b) NR/GC. 

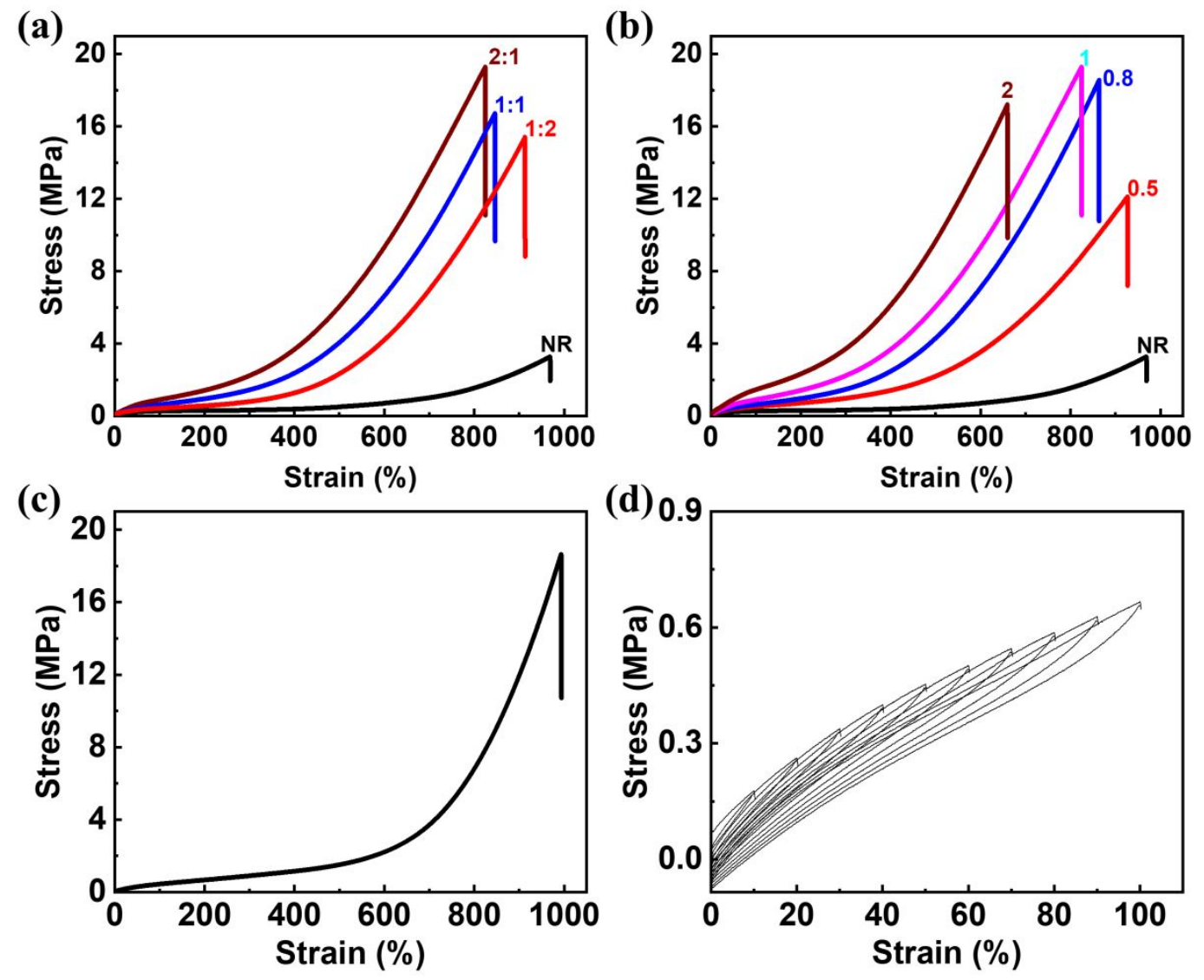
Figure S7. Stress-strain curves (a) of NR/GC (The filler content is $1 \mathrm{wt} \%$ but the ratio of $\mathrm{GO}$ and $\mathrm{NC}$ is different.), (b) $\mathrm{NR} / \mathrm{GC}-2: 1$ with loading from 0.5 to $2 \mathrm{wt} \%$, (c) stress-strain curve of NR/S, (d) cyclic stress-strain curve of NR.
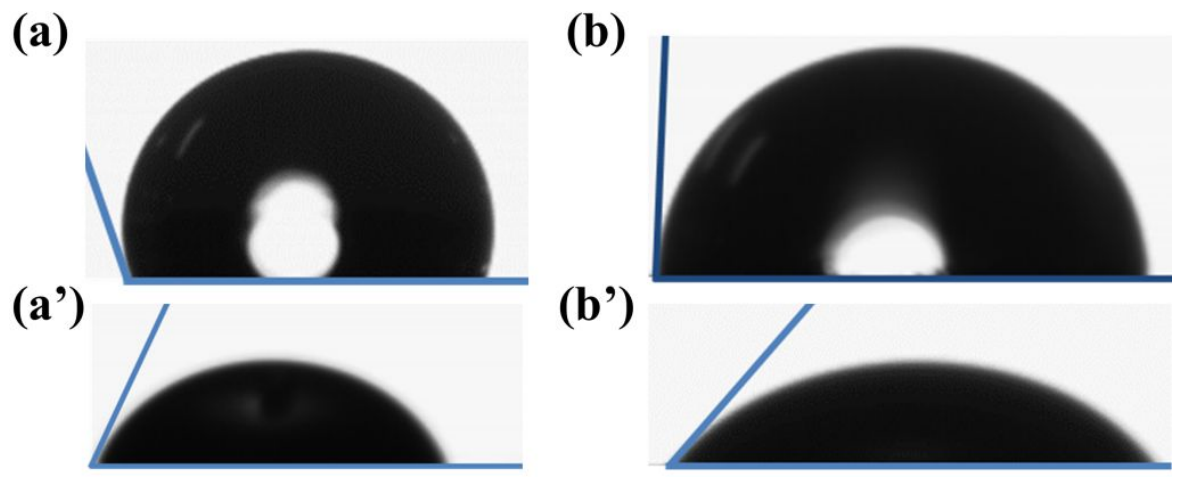

Figure S8. The contact angle between the solids film (NR/S, NR/GC) and water $(a \sim b)$ and diiodomethane $\left(a^{\prime} \sim b^{\prime}\right)$. 


\section{Table S1}

Quantitative predictors for interfacial interaction in composites.

\begin{tabular}{|c|c|c|c|c|c|}
\hline samples & $\begin{array}{c}\boldsymbol{\theta}\left(\mathrm{H}_{2} \mathrm{O}\right) \\
\left({ }^{\mathbf{0}}\right)\end{array}$ & $\begin{array}{c}\boldsymbol{\theta}\left(\mathrm{CH}_{2} \mathbf{I}_{2}\right) \\
\left({ }^{\mathbf{o}}\right)\end{array}$ & $\begin{array}{c}\gamma^{\mathrm{d}} \\
(\mathrm{mN} / \mathrm{m})\end{array}$ & $\begin{array}{c}\gamma^{p} \\
(\mathrm{mN} / \mathbf{m})\end{array}$ & $\begin{array}{c}\gamma^{\text {surace }} \\
(\mathrm{mN} / \mathrm{m})\end{array}$ \\
\hline $\mathbf{N R} / \mathbf{S}$ & 108.5 & 64.7 & 26.9 & 0.01 & 26.9 \\
\hline NR/GC & 87.6 & 48.7 & 32.6 & 2.4 & 35.1 \\
\hline
\end{tabular}




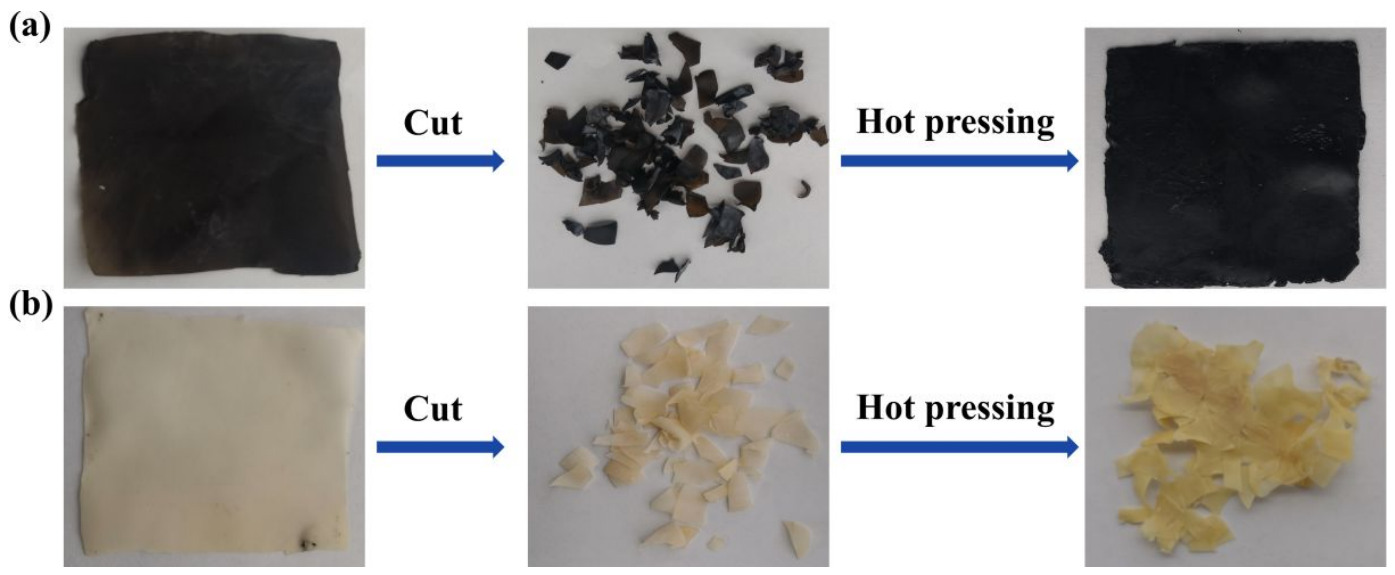

Figure S9. Recycling of (a) NR/GC films and (b) NR/S films. 